\title{
Daño arterial asociado a la enfermedad renal crónica: evaluación mediante técnicas de laboratorio no invasivo en pacientes hemodializados
}

\author{
FRANCISCO ESPINOZA ${ }^{1}$, SONIA KUNSTMANN ${ }^{1,2}$, ALVARO URZÚA ${ }^{1}$, \\ LUIS MICHEA $^{1, a}$, ELISA T. MARUSIC ${ }^{1}$, ANTONIO VUKUSICH $^{1,3}$
}

\section{Assesment of arterial damage by noninvasive peripheral arterial tonometry in non-diabetic hemodialysis patients}

Background: Hemodialysis patients (HD) display high rates of cardiac disease and mortality. The cardiovascular morbidity and mortality of HD patients is attributable in a significant proportion to endothelial dysfunction, arterial stiffness, and vascular calcifications. Aim: To measure vascular reactivity in HD subjects and compare them with healthy volunteers. Material and Methods: Forty eight non diabetic patients aged $58 \pm 4.6$ years (29 males) on hemodialysis for a mean lapse of 4.8 years were studied. Arterial stiffness was measured in the radial artery. Pulse wave velocity was measured by noninvasive peripheral arterial tonometry in carotid and femoral arteries. Endothelial function was assessed, measuring reactive hyperemia response after a 5 min period of ischemia. As a control, all values were also measured in age and gender-matched healthy volunteers. Results: Arterial stiffness was significantly higher in HD patients than controls (23.9 \pm 3.3 and $18.4 \pm 3.4 \%$ respectively, $p<0.05)$. HD subjects had an increased pulse wave velocity $(10.0 \pm 0.8$ and $7.6 \pm 0.9 \mathrm{~m} / \mathrm{s}$ respectively, $p<0.05)$. A reduction in the change in pulse amplitude pressure, as a measure of arterial dysfunction, was only observed in male patients $(1.7 \pm 0.4$ and $2.7 \pm 0.4$ respectively $p<0.01)$. Conclusions: Noninvasive assessment of peripheral vascular function may be useful for the identification of patients at risk for late cardiac events.

(Rev Med Chile 2012; 140: 153-160).

Key words: Diagnostic techniques, cardiovascular; Renal insufficiency; Vascular diseases.

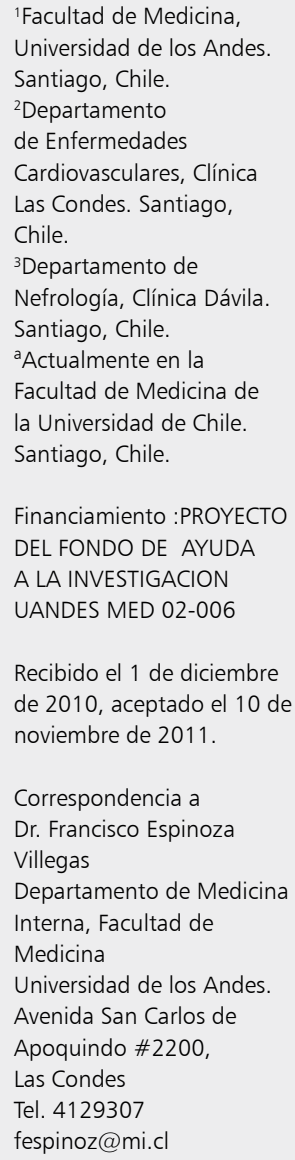

\section{I} a enfermedad renal crónica (ERC) se asocia a enfermedad cardiovascular, por lo cual en la ERC avanzada el riesgo de sufrir un evento cardiovascular es 20 a 100 veces superior al de la población general ${ }^{1}$. La anemia, la hipertensión secundaria, la dislipidemia y alteraciones del producto calcio-fósforo, generan mecanismos de oxidación, inflamación y disfunción endotelial que provocan daño arterial ${ }^{2,3}$.
Este último concepto ha sido reconocido como factor independiente de morbi-mortalidad cardiovascular y representa alteraciones estructurales y dinámicas de los vasos sanguíneos expresadas por aumentos de la rigidez arterial y disfunción endotelial, respectivamente ${ }^{4}$. Ambos parámetros se correlacionan con enfermedades cardiovasculares ${ }^{5,6} \mathrm{y}$ han demostrado su utilidad en otras poblaciones de riesgo ${ }^{3,7,8}$. El índice de aumento de la onda de 
pulso aórtica y la velocidad de conducción de onda de pulso permiten cuantificar la rigidez arterial ${ }^{9}$. La hiperemia digital reactiva post-isquemia estima la disfunción endotelial.

Diversos estudios han demostrado que la velocidad de onda de pulso es un predictor independiente de mortalidad ${ }^{4}$. El tamaño de la onda de pulso en la raíz aórtica aumenta como resultado de la reflexión de la onda de pulso en la periferia del árbol arterial, que viaja en forma retrógrada hacia la raíz de la aorta. Por tanto, aumentos de la rigidez arterial causan aumentos de la amplitud de la onda de pulso.

Por otro lado, la disfunción endotelial precede a los cambios morfológicos de la rigidez arterial y ocurriría por una disminución en la biosíntesis y biodisponibilidad de vasodilatadores como el óxido nítrico. La evidencia indica que la disfunción endotelial participa en el daño cardiovascular de la $\mathrm{ERC}^{2,3}$. La hiperemia digital reactiva postisquemia, estimada como el cambio en la amplitud de la onda de pulso digital luego de un período de isquemia, es un nuevo método para estimar la disfunción endotelial ${ }^{10}$. Un reciente estudio transversal, en la tercera generación de participantes del estudio de Framingham, demostró que los cambios de la hiperemia digital están asociados a factores de riesgo cardiovascular tradicionales, sugiriendo su potencial aplicabilidad en el contexto clínico ${ }^{11}$.

El objetivo del estudio es analizar las características del daño arterial en la ERC avanzada de una muestra de pacientes no diabéticos, mediante técnicas vasculares no invasivas.

\section{Pacientes y Métodos}

\section{Pacientes}

Se incluyeron pacientes que se encontraban en hemodiálisis por al menos 12 meses, sin función renal residual y mayores de 18 años. Fueron excluidos aquellos pacientes con hipertensión refractaria a tratamiento, diabéticos, con antecedentes de cáncer, daño hepático crónico, Raynaud o con imposibilidad técnica de realizar los exámenes: fístula arteriovenosa bilateral, fibrilación auricular y pulso radial u onda de pulso digital no identificable. La muestra quedó constituida por 48 pacientes (grupo HD) que se compararon con 55 individuos con menos de dos (0-1) factores de riesgo cardiovascular (grupo control), pareados por edad y sexo.
En ambos grupos se determinó: el índice de aumento de la onda de pulso (AIx), la velocidad de la onda de pulso (PWV) y la hiperemia digital reactiva postisquemia (HDRPI). Las mediciones tonométricas digitales y radiales se realizaron en el brazo sin FAV, el día siguiente a la diálisis de media semana.

El estudio fue aprobado por el Comité de Ética de la Facultad de Medicina de la Universidad de los Andes.

\section{Métodos de laboratorio no invasivo}

Se analizó la forma de la onda de pulso de la arteria radial mediante el tonómetro SPC-301 (Millar Instruments). Los datos promediados de 10 registros consecutivos fueron considerados para el cálculo del índice de aumento de la onda de pulso aórtica (AIx), mediante el programa computacional SphygmoCor 7.01 (AtCor Medical, Australia), normalizado para una frecuencia cardiaca de 75 latidos por minuto (AIx@75) (Figura 1).

La velocidad de conducción de onda de pulso (PWV) fue determinada mediante registro de la onda de pulso carotídea y femoral, utilizando un transductor piezoeléctrico conectado al sistema de adquisición de datos PowerLab 4/25T (AD Instruments, Australia). El pulso carotídeo se midió en la base del cuello en su cara lateral izquierda y el femoral en la zona inguinal izquierda. El ECG y las ondas de pulso fueron registrados y analizados con el programa Charts5 for Windows (AD Instruments, Australia). El tiempo entre la despolarización ventricular y el inicio de la onda de pulso carotídea o femoral fue determinado mediante el cálculo de la diferencia de tiempo entre el registro de la onda $\mathrm{R}$ del electrocardiograma y la base de las ondas de pulso. La distancia recorrida por la onda de pulso fue calculada mediante la diferencia de la distancia entre ambas zonas, restando la distancia entre la carótida y el hueco supraesternal. La PWV fue calculada como:

$$
\operatorname{PWV}=\frac{\Delta \mathrm{d}}{\Delta \mathrm{t}}=\frac{(\mathrm{dCF})-(\mathrm{dCSE})}{(\mathrm{tF}-\mathrm{tC})}
$$

dCF: distancia carótida-femoral. dCSE: distancia carótida-hueco supraesternal (m). tF y tC:tiempo en que llega la onda a la femoral y la carótida (s), respectivamente (Figura 2).

La función endotelial fue evaluada mediante un tonómetro arterial para medir la amplitud de 


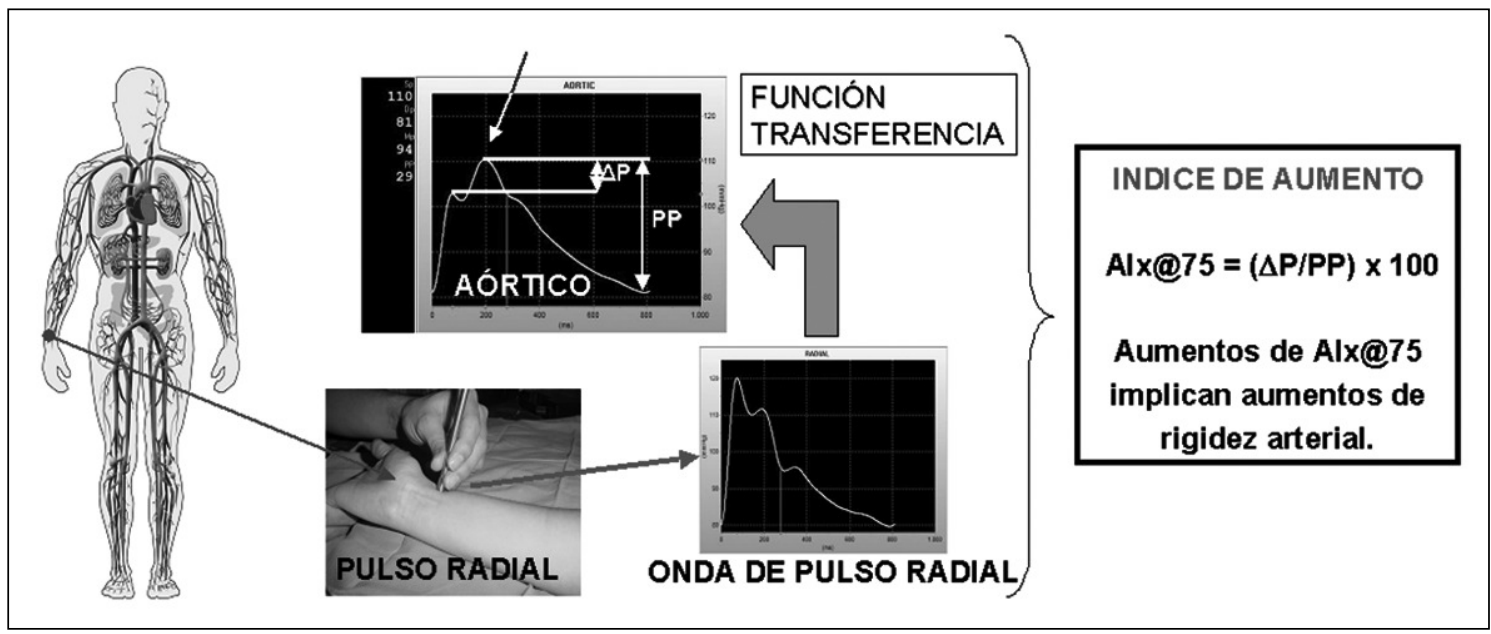

Figura 1. Tonometría de pulso para estimación del Indice de Aumento.

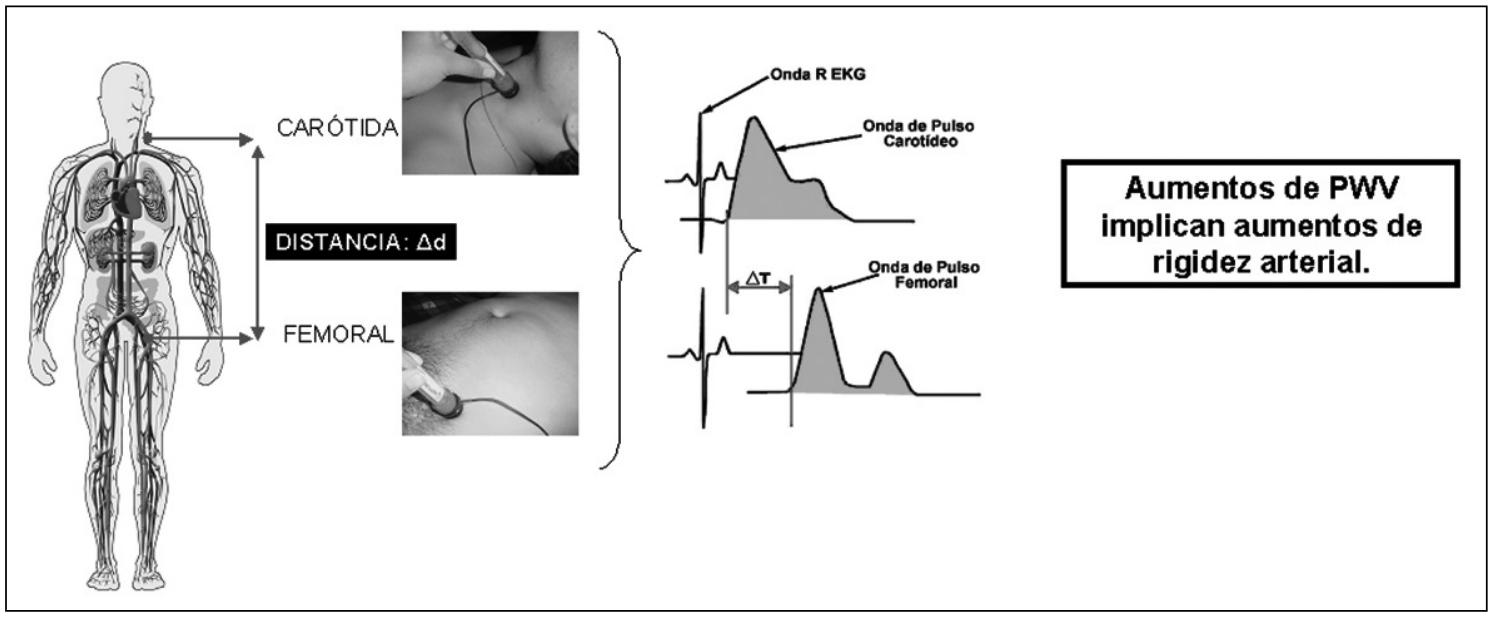

Figura 2. Velocidad de onda de pulso

volumen del pulso digital del dedo índice. Éste se coloca en un sistema cerrado, presurizado e inflable. Esto permite aplicación de presiones constantes y homogéneas, lo que incrementa la sensibilidad del registro y previene el aumento de la capacitancia venosa que ocasiona un reflejo vasoconstrictor venoarteriolar. Los cambios de amplitud se registran mediante un transductor a un computador. La tonometría se realiza con el paciente en posición supina, en ambiente termoneutral, midiéndose la presión arterial basal. Primero, se establece un tiempo de equilibrio de 3 minutos (tiempo preisquemia), luego se infla el manguito $60 \mathrm{mmHg}$ por sobre la presión sistólica en el brazo estudiado. El tiempo de isquemia es de 5 minutos, y al cabo de éste, se desinfla el manguito y se continúa registrando por 2 minutos más. El cálculo del cambio en la amplitud de volumen de pulso luego de la hiperemia reactiva se realiza mediante el promedio de 1 minuto de registro después de 1 minuto postisquemia, dividido por el promedio de registro de los 3 minutos preisquemia. La razón de usar el período de tiempo postisquemia señalado es debido a que refleja de mejor manera la función endotelial coronaria ${ }^{10}$ (Figura 3 ).

Control del sesgo: Los operadores fueron dos para la AIx y PWV y uno, ciego respecto del resultado anterior, para la medición de HRDPI. Fueron entrenados aceptándose en el caso de AIx índices de calidad de registro (similitud entre las ondas 


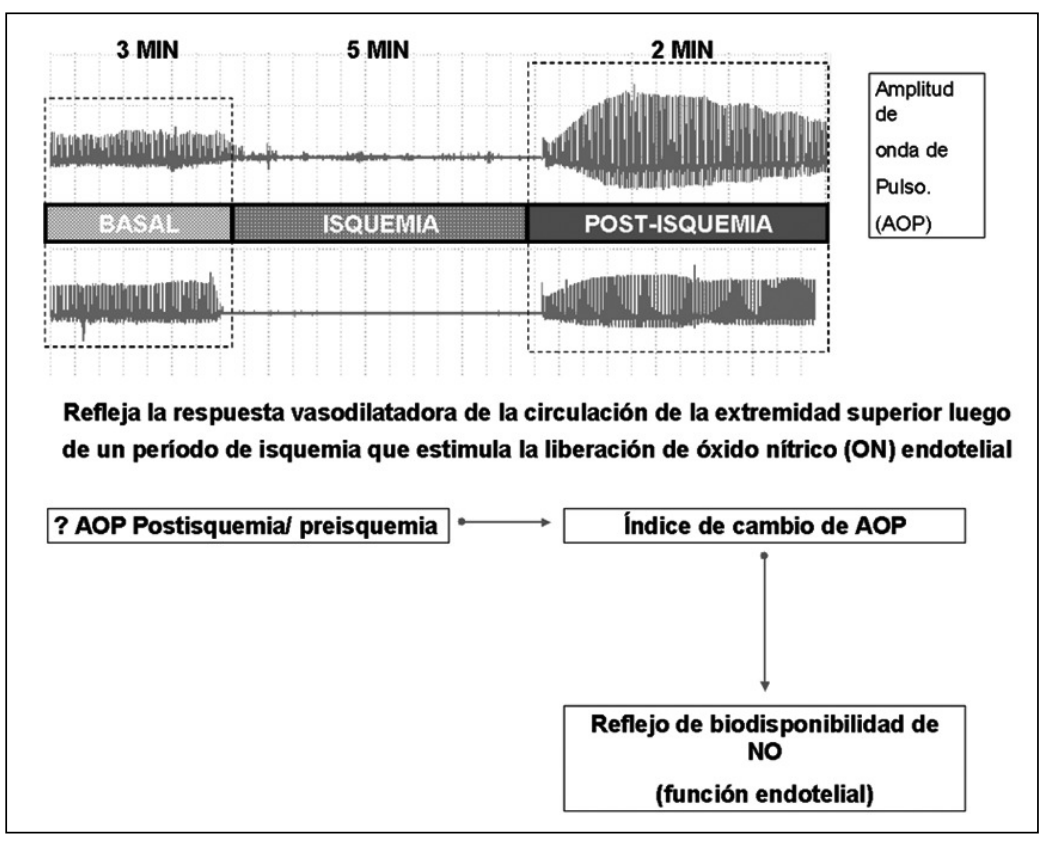

Figura 3. Hiperemia digital reactiva post isquemia. registradas) mayores a $85 \%$. Múltiples estudios demuestran un alto grado de reproducibilidad y repetibilidad ${ }^{12-14}$. Las diferencias interobservador reportadas son entre 0,23 y $0,4+2,4 \%$. Las diferencias intraobservador son de $0,5+0,9 \%$.

Las variables continuas se describieron mediante la mediana, promedio y desviación estándar. Se docimó la normalidad de las variables continuas mediante el test de Shapiro-Wilk y, en consecuencia las variables que resultaron tener distribución normal fueron comparadas mediante el test t-Student para muestras independientes y en caso de descartarse la normalidad fueron comparadas mediante el test de Mann-Whitney.

\section{Resultados}

\section{Características de la población}

La población en estudio estuvo compuesta por 48 pacientes con ERC en HD. El 54\% de ellos eran hombres y el promedio de tiempo en HD fue de 4,8 años. En la Tabla 1 se incluyen las características generales del grupo HD y del grupo control. No se obtuvieron diferencias significativas en edad o sexo. La prevalencia de hipertensión arterial en el grupo de dializados fue mayor ( $85 \%$ vs $45 \%$ ). En ambos grupos hubo un porcentaje significativo de individuos con PA no controlada (> 140/90), sin embargo,
$16 \%$ de los sujetos del grupo control estaba sin tratamiento farmacológico. En cuanto a la terapia antihipertensiva los pacientes dializados recibían más bloqueadores de calcio ( $45 \%$ vs $27 \%$ ) y beta bloqueadores $(26 \%$ vs $7 \%)$ y menos inhibidores de la enzima convertidora ( $21 \%$ vs $45 \%)$, con una diferencia estadísticamente significativa $(\mathrm{p}<0,001)$.

\section{Parámetros de daño arterial}

Se compararon indicadores de rigidez arterial entre el grupo HD y el grupo control. Se observó un índice de aumento significativamente mayor en el grupo de dializados que en los controles $(23,94$ $\pm 3,3 \%$ vs $18,35 \pm 3,4 \%, \mathrm{P}<0,05$ ) (Figura $4 \mathrm{~A}$ ). La velocidad de onda de pulso también resultó ser significativamente mayor en $\mathrm{HD}(9,95 \pm 0,78 \mathrm{~m} / \mathrm{s}$ vs $7,59 \pm 0,69 \mathrm{~m} / \mathrm{s}, \mathrm{P}<0,05$ ) (Figura $4 \mathrm{~B}$ ).

La función endotelial fue evaluada mediante hiperemia digital reactiva post isquemia. El índice de cambio en la amplitud de la onda de pulso se midió en la población de hemodializados y en los controles, observándose una disminución significativa en el índice de pacientes hombres HD: 1,66 $\pm 0,38$ vs $2,66 \pm 0,41, P<0,001$, (Figura $5 \mathrm{~A}$ ). No se observó lo mismo en la población de mujeres $\mathrm{HD}$, las cuales presentaron un índice de cambio similar a mujeres controles e inferior al de los hombres (Figura 5B). 
Laboratorio vascular no invasivo en hemodializados - F. Espinoza et al

Tabla 1. Características clínicas generales de los pacientes en estudio y controles

\begin{tabular}{|c|c|c|c|}
\hline & Pacientes & Controles & Diferencia (p) \\
\hline Número de individuos (n) & 48 & 55 & \\
\hline Edad (años) & $58 \pm 4,6$ & $57 \pm 5,2$ & 0,67 \\
\hline Hombres, n (\%) & $29(54 \%)$ & $31(56 \%)$ & 0,48 \\
\hline $\mathrm{IMC}\left(\mathrm{kg} / \mathrm{m}^{2}\right)$ & $23,3 \pm 1,5$ & $25,2 \pm 2,7$ & 0,21 \\
\hline Presión arterial > 140/90, n (\%) & $19(35 \%)$ & $18(32 \%)$ & 0,11 \\
\hline Bloqueadores de calcio (\%) & $22(45 \%)$ & $15(27 \%)$ & $<0,001$ \\
\hline Inhibidores de enzima convertidora (\%) & $10(21 \%)$ & $25(45 \%)$ & $<0,001$ \\
\hline Antagonistas del receptor de angiotensina (\%) & $4(8 \%)$ & $3(5 \%)$ & 0,14 \\
\hline Beta bloqueadores & $12(26 \%)$ & $4(7 \%)$ & $<0,01$ \\
\hline Sin tratamiento antihipertensivo & $0(0 \%)$ & $8(16 \%)$ & $<0,001$ \\
\hline Presión Arterial Sistólica (mmHg) & $146 \pm 13$ & $141 \pm 10$ & 0,19 \\
\hline Presión Arterial Diastólica (mmHg) & $86 \pm 8$ & $82 \pm 7$ & 0,38 \\
\hline Frecuencia Cardiaca (lat/min) & $63 \pm 7$ & $76 \pm 9$ & 0,15 \\
\hline Dislipidemia, n (\%) & $13(24 \%)$ & $10(18 \%)$ & $<0,05$ \\
\hline Tabaquismo, n (\%) & $6(11 \%)$ & $7(12 \%)$ & 0,41 \\
\hline
\end{tabular}
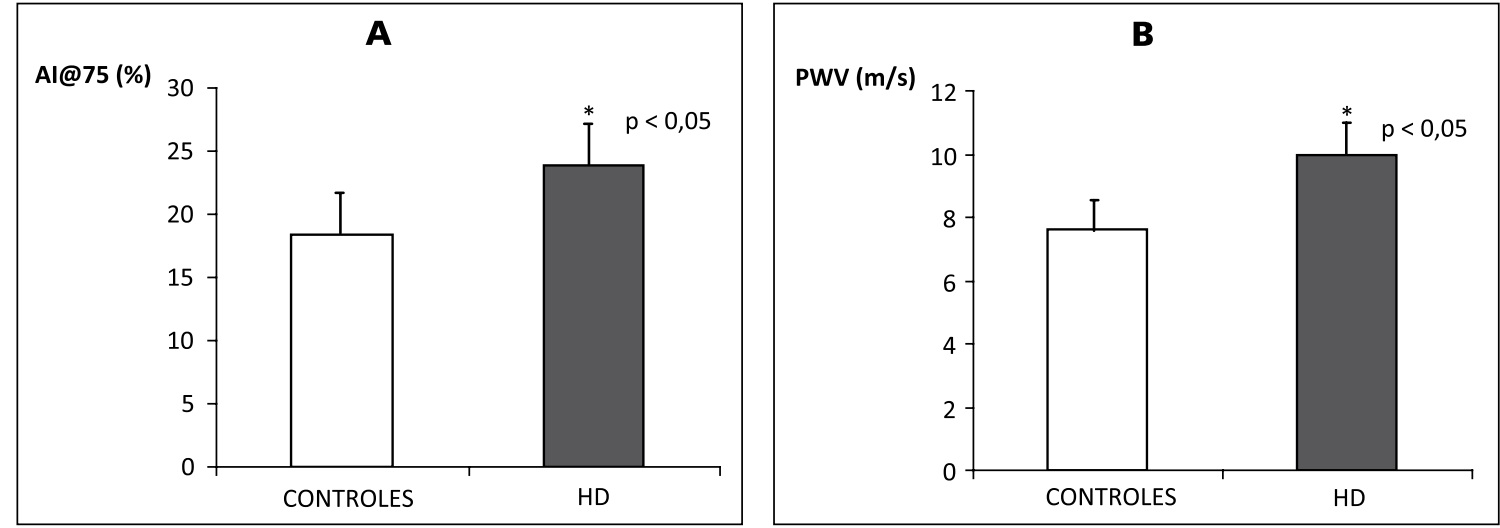

Figura 4. (A) Índice de aumento (Axl@75) y (B) Velocidad de onda de pulso (PWV). Comparación de pacientes vs controles.
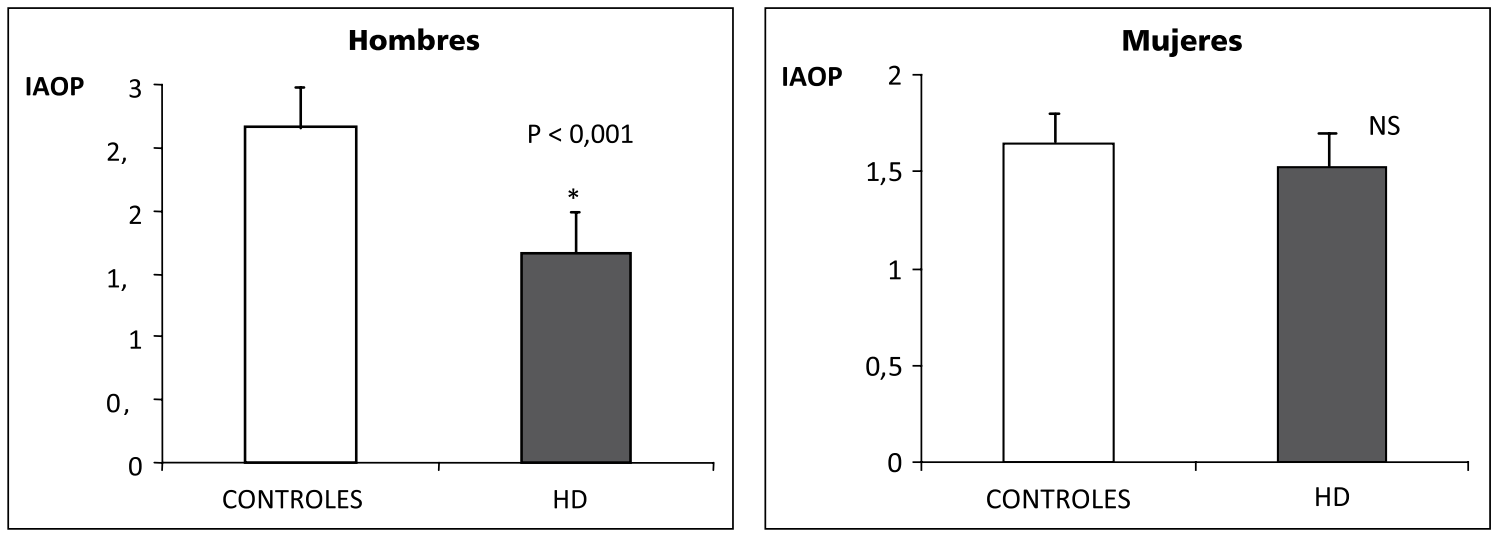

Figura 5. Hiperemia digital reactiva post-isquemia. Comparación por género entre pacientes y controles. 


\section{Discusión}

El daño arterial tiene dos componentes: la rigidez arterial y la disfunción endotelial. La primera define el endurecimiento de la pared arterial y aumenta con la edad, hipertensión, diabetes, dislipidemia y obesidad ${ }^{15,16}$. Se puede estimar mediante la velocidad de la onda de pulso, que se considera como el "gold standard"17. Como la aorta es el principal componente de la elasticidad arterial, se utiliza la medición carótido-femoral. A mayor velocidad, mayor es la rigidez y menor la distensibilidad. Una evaluación de vasos de menor calibre se puede obtener mediante el índice de aumento. Con este método se mide la amplitud de la onda de pulso. La medición permite el cálculo de la onda de pulso a nivel aórtico, utilizando una función de transferencia validada por estudios invasivos ${ }^{18-20}$. A partir de la onda de pulso aórtica es posible calcular el llamado índice de aumento, que es la diferencia entre los componentes $\mathrm{P} 2$ y $\mathrm{P} 1$, expresados como porcentaje de la presión diferencial (sistólica-diastólica). Si el valor es positivo (P2 mayor a P1) representaría una mayor rigidez arterial, debido a que $\mathrm{P} 2$ representa el reflejo de la onda sobre la pared arterial. Así a mayor componente $\mathrm{P} 2$, mayor reflejo y por tanto, mayor rigidez de la pared arterial. Nuestros resultados demuestran que en la población HD existe una mayor rigidez arterial, expresada por una diferencia significativa en el aumento de la velocidad de la conducción de la onda de pulso y del índice de aumento. El presente estudio se realizó en pacientes HD no diabéticos, para descartar la implicancia de la diabetes como factor asociado a daño arterial.

Estudios recientes, en pacientes con ERC avanzada y diabéticos demostraron correlación significativa entre el incremento de velocidad de onda de pulso e índice de aumento y severidad de enfermedad coronaria o muerte cardiovascular ${ }^{21,22}$. El estudio CRIC (Chronic Renal Insufficiency Cohort) mostró una asociación positiva entre velocidad de onda y factores de riesgo generales como edad, glicemia, presión arterial media, sexo masculino y una asociación negativa con función renal $^{23}$. Sin embargo, existe controversia si el grado de avance de la ERC pudiera explicar la severidad de la rigidez arterial. Tetzner et al analizaron 3 grupos de pacientes con ERC en etapas 2, 4 y 5 , no pudiendo demostrar una correlación entre velocidad de filtración glomerular y rigidez arterial ${ }^{24}$.
Los pacientes del grupo HD tenían un mayor uso de bloqueadores de calcio y beta bloqueadores. Al respecto, no existen evidencias de que una terapia antihipertensiva específica pueda afectar el resultado de las pruebas de rigidez.

Diferentes estudios se han centrado en la medición de el espesor de íntima media carotídea (EIMC). ¿Son equivalentes velocidad de onda o índice de aumento con el EIMC en cuanto a su significado o correlación entre sí o con eventos cardiovasculares? A pesar de que ambos son predictores independientes de eventos $\mathrm{CV}$ y se desarrollan en paralelo, la respuesta parece ser no. Los factores de riesgo específicos mencionados anteriormente para rigidez arterial no son los mismos. No se ha demostrado que la velocidad de onda se correlacione con EIMC. Shoji et $\mathrm{a}^{25}$, en una cohorte de 423 hemodializados seguida por 70 meses, compararon la correlación entre rigidez arterial-EIMC y su asociación con eventos cardiovasculares. Los resultados mostraron que parámetros de rigidez predecían mortalidad independiente del grosor de la pared arterial, revelando distinto tipo de injuria vascular asociada a la ERC. Recientemente, Vukusich et $\mathrm{al}^{26}$ han demostrado en un ensayo clínico controlado, realizados en hemodializados no diabéticos, que la inhibición farmacológica de aldosterona durante 24 meses es capaz de reducir significativamente el EIMC. Sería interesante conocer si espironolactona es capaz de revertir el aumento de la rigidez arterial , como su potencial efecto en la disfunción endotelial.

La evaluación indirecta de la biodisponibilidad de óxido nítrico mediante técnicas no invasivas, se centra en la respuesta vasodilatadora de los vasos arteriales al estimular su producción mediante isquemia. El método más conocido es la medición ecográfica del calibre de la arteria braquial pre y post isquemia (oclusión del vaso). En el presente trabajo se examinó el cambio en la amplitud de la onda de pulso medida en la circulación digital también siguiendo el principio de la isquemia como estimulador de la liberación de óxido nítri$\mathrm{CO}^{20,27}$. La disfunción endotelial, representa una vía común de expresión que induce una alteración del metabolismo oxidativo, inflamación arterial y en consecuencia anomalías de la reactividad arterial. También es reconocida su participación como factor de riesgo independiente de enfermedad cardiovascular. La hiperemia reactiva digital se encuentra disminuida en niños diabéticos y en pacientes con 
microvasculopatía ${ }^{10,28}$. Un tipo de betabloqueador, el nevibolol, con acciones sobre receptores $\beta 3$, puede aumentar la biodisponibilidad de óxido nítrico. También este efecto ha sido reportado para inhibidores de la enzima convertidora y antagonistas de angiotensina ${ }^{29}$. En nuestro estudio, ningún paciente usó nevibolol y tampoco hay reportes de hiperemia reactiva digital y el fármaco en cuestión.

Los resultados obtenidos en el presente estudio sobre función endotelial en pacientes $\mathrm{HD}$, señalan importantes diferencias de acuerdo al sexo, siendo sólo el grupo de hombres el que presenta disfunción comparado con el grupo control de similar edad. La biodisponibilidad reducida de NO sería uno de los mayores factores involucrados en la disfunción endotelial asociada a la enfermedad renal debido mayormente al aumento del estrés oxidativo en la pared vascular ${ }^{1,30,31}$ alteración que según nuestro resultados ocurriría preferencialmente en el sexo masculino.

Una posible explicación del fenómeno anterior puede venir de hallazgos en modelos animales. Existe una diferencia de género en cuanto a la biodisponibilidad de óxido nítrico que parece depender de 2 fenómenos centrales:

a) Se ha determinado que niveles bajos de adiponectina disminuyen la producción de óxido nítrico. La hipoadiponectemia se asocia a insulinoresistencia y explicaría en parte la disfunción endotelial en diabetes, sin embargo, parece ser que existe una diferencia constitucional de género y que las ratas de sexo masculino tienen intrínsecamente niveles más bajos de adiponectina ${ }^{32,33}$. A pesar de que en la enfermedad renal los niveles de adiponectina suelen estar elevados, la alta concentración de cistatina $\mathrm{C}$ anula los efectos protectores de ésta ${ }^{34}$.

b) La dimetil-arginina asimétrica (DMAA) es un producto de la metilación proteica y es un inhibidor endógeno de la óxido nítrico sintetasa endotelial. Las mujeres tienen un incremento en la concentración de éste producto mucho más tardío que los hombres y por tanto, éste sería otro factor que podría explicar la disfunción endotelial más severa y precoz en el sexo masculino. La DMAA en enfermedad renal crónica se encuentra en concentraciones altas y recientemente Schepers et al. Han descrito un rol porinflamatorio en ERC, activando NF- $\kappa \mathrm{B}$, lo que lleva al aumento de la expresión de IL-6 y TNF alfa ${ }^{35}$. Todo lo anterior podría contribuir a exacerbar las diferencias de género planteadas.
En resumen, el presente estudio nos muestra el empleo de técnicas no invasivas para determinar el daño vascular y microvascular en una muestra de conocido aumento de riesgo cardiovascular, como es la ERC. En un futuro trabajo se podría examinar la evolutividad de éstos parámetros siguiendo a los pacientes a medida que progresa la insuficiencia renal y conocer si se asocian a un mayor riesgo cardiovascular en un período de tiempo. Las tres técnicas de laboratorio vascular no invasivo, simples y reproducibles, permitieron inferir rigidez arterial y disfunción endotelial.

\section{Referencias}

1. Schiffrin E, Lipman M, Mann J. Effects of chronic kidney disease on cardiovascular system. Circulation 2007; 116: 85-97.

2. Vaziri ND, Oveisi F, Ding YX. Role of increased oxygen free radical activity in the pathogenesis of uremic hypertension. Kidney Int 1998; 53: 1748-54.

3. Vaziri ND, Ni Z, Oveisi F, Liang K, Pandian R. Enhanced nitric oxide inactivation and protein nitration by reactive oxygen species in renal insufficiency. Hypertension 2002; 39: 135-41.

4. Weber T, Auer J, O’Rourke MF, Kvas E, Lassnig E, Berent $\mathrm{R}$, et al. Arterial stiffness, wave reflections, and the risk of coronary artery disease. Circulation. 2004;109: 184-9.

5. London GM, Blacher J, Pannier B, Guerin AP, Marchais SJ, Safar ME. Arterial wave reflections and survival in end-stage renal failure. Hypertension 2001; 38: 434-8.

6. Safar ME, Blacher J, Pannier B, Guerin AP, Marchais SJ, Guyonvarc'h PM, et al. Central pulse pressure and mortality in end-stage renal disease. Hypertension 2002; 39: 735-8.

7. Franklin SS, Khan SA, Wong ND, Larson MG, Levy D. Is pulse pressure useful in predicting risk for coronary heart Disease? The Framingham heart study. Circulation 1999; 100: 354-60.

8. Blacher J, Asmar R, Djane S, London GM, Safar ME. Aortic pulse wave velocity as a marker of cardiovascular risk in hypertensive patients. Hypertension 1999; 33: 1111-7.

9. Corretti M, Anderson T, Benjamin E, Celermajer D, Charbonneau F, Creager MA, et al. Guidelines for the ultrasound assessment of endothelial-dependent flowmediated vasodilatation of the brachial artery. J Am Coll Cardio 2002; 39: 257 - 65.

10. Bonetti P, Pumper G, Higano S, Holmes DR Jr, Kuvin JT, Lerman A. Noninvasive identificacion of patients with early coronary atherosclerosis by assessment of digital 
reactive hyperemia. J Am Coll Cardio 2004; 44: 2137-41.

11. Celermajer D. Reliable endothelial function testing: at our fingertips. Circulation 2008; 117: 2428-30.

12. Wilkinson IB, Fuchs SA, Jansen IM, Spratt JC, Murray GD, Cockcroft JR, et al. Reproducibility of pulse wave velocity and augmentation index measured by pulse wave analysis. J Hypertension 1998; 16: 2079-84.

13. Seibenhofer A, Kemp CRW, Sutton J, Williams B. The reproducibility of central aortic blood pressure measurements in healthy subjects using applanation tonometry and sphygmocardiography. J Hum Hypertens 1999; 13: 625-9.

14. Filipovsky J, Svobodova V, Pecan L. Reproducibility of radial pulse wave analysis in healthy subjects. J Hypertension 2000; 18: 1033- 40.

15. Safar ME, Levy BI, Struijker-Boudier H. Current perspectives on arterial stiffness and pulse pressure in hypertension and cardiovascular diseases. Circulation 2003; 107: 2864-9.

16. Woodman RJ, Watts GF. Measurement and application of arterial stiffness in clinical research: focus on new methodologies and diabetes mellitus. Med Sci Monit 2003; 9: 81-9.

17. Tsuchikura S, Shoji T, Kimoto E, Shinohara K, Hatsuda $\mathrm{S}$, Koyama H, et al. Central versus peripheral arterial stiffness in association with coronary, cerebral and peripheral arterial disease. Atherosclerosis 2010; 211 (2): 480-5.

18. Vaudo G, Schillaci G, Evangelista F, Pasqualini L, Verdecchia P, Mannarino E. Arterial wall thickness at different sites and its association with left ventricular hypertrophy in newly diagnosed essential hypertension. Am J Hypertens 2000; 13: 324 - 31.

19. Chen CH, Nevo E, Fetics B, Pak PH, Yin FC, Maughan WL, Kass DA. Estimation of central aortic pressure waveform by mathematical transformation of radial tonometry pressure. Validation of generalizated transfer function. Circulation 1997; 95: 1827-36.

20. Hope SA, Tay DB, Meredith IT, Cameron JD. Comparison of generalized and gender-specific transfer functions for the derivation of aortic waveforms. Am J Heart Circ Physiol 2002; 283: 150-6.

21. Khoshdel AR, Carney SL, Trevillian P, Gillies A. Evaluation of arterial stiffness and pulse wave reflection for cardiovascular risk assessment in diabetic and nondiabetic kidney transplant recipients. Iran J Kidney Dis 2010; 4 (3): 237-43.

22. Verbeke F, Van Biesen W, Honkanen E, Wikström B, Jensen PB, Krzesinski JM, et al. Prognostic Value of Aortic Stiffness and Calcification for Cardiovascular Events and Mortality in Dialysis Patients: Outcome of the Calcifi- cation Outcome in Renal Disease (CORD) Study. Clin J Am Soc Nephrol 2011; 6 (1): 153-9.

23. Townsend RR, Wimmer NJ, Chirinos JA, Parsa A, Weir M, Perumal K, et al. Aortic PWV in chronic kidney disease: a CRIC ancillary study. Am J Hypertens 2010; 23 (3): 282-9.

24. Tetzner F, Scholze A, Wittstock A, Zidek W, Tepel M. Impaired vascular reactivity in patients with chronic kidney disease. Am J Nephrol 2008; 28 (2): 218-23.

25. Shoji T, Maekawa K, Emoto M, Okuno S, Yamakawa T, Ishimura E, et al. Arterial stiffness predicts cardiovascular death independent of arterial thickness in a cohort of hemodialysis patients. Atherosclerosis 2010; 210 (1): 145-9.

26. Vukusich A, Kunstmann S, Varela C, Gainza D, Bravo S, Sepulveda D, et al. A randomized, double-blind, placebo-controlled trial of spironolactone on carotid intima-media thickness in nondiabetic hemodialysis patients. Clin J Am Soc Nephrol 2010; 5 (8): 1380-7.

27. Nohria A, Gerhard-Herman M, Creager MA, Hurley S, Mitra D, Ganz P. Role of nitric oxide in the regulation of digital pulse volume amplitude in humans. J Appl Physiol 2006; 101: 545-8.

28. Haller MJ, Stein J, Shuster J, Theriaque D, Silverstein J, Schatz DA, et al. Peripheral artery tonometry demonstrates altered endothelial function in children with type 1 diabetes. Pediatr Diabetes 2007; 8: 193-8.

29. Korkmaz H, Onalan O. Evaluation of endothelial dysfunction: flow-mediated dilation. Endothelium 2008; 15 (4): 157-63.

30. Al Suwaidi J, Hamasaki S, Higano ST, Nishimura RA, Holmes DR. Long-term follow-up of patients with mild coronary artery disease and endothelial dysfunction. Circulation 2000; 101: 948-54.

31. Siekmeier R, Grammer T, März W. Role of Oxidants, Nitric Oxide and Asymmetric Dimethylarginine in Endothelial Function. J Cardiovasc Pharmacol Ther 2008; 13(4): 279-97.

32. Tan KC, Xu A, Chow WS, Lam MC, Ai VH, Tam SC, et al. Hypoadiponectinemia is associated with impaired endothelium-dependent vasodilatation. J Clin Endocrinol Metab 2004; 89: 765-69.

33. Takenouchi Y, Kobayashi T, Taguchi K, Matsumoto T, Kamata K. Possible Involvement of Akt Activity in Endothelial Dysfunction in Type 2 Diabetic Mice. J Pharmacol Sci 2008; 106: 600-8.

34. Komura N, Kihara S, Sonoda M, Maeda N, Tochino Y, Funahashi T, et al. Increment and impairment of adiponectin in renal failure. Cardiovasc Res. 2010; 86: 471-7.

35. Schepers E, Barreto DV, Liabeuf S, Glorieux G, Eloot S, Barreto FC, et al. Symmetric Dimethylarginine as a Proinflammatory Agent in Chronic Kidney Disease. Clin J Am Soc Nephrol 2011 Aug 4. [Epub ahead of print]. 